\title{
A synchronous papillary and follicular thyroid carcinoma presenting as a large toxic nodule in a female adolescent
}

\author{
Joke Van Vlaenderen ${ }^{1 *}$ D, Karl Logghe ${ }^{2}$, Eva Schiettecatte ${ }^{3}$, Hubert Vermeersch ${ }^{4}$, Wouter Huvenne ${ }^{4}$, \\ Kathleen De Waele ${ }^{1}$, Hanne Van Beveren ${ }^{5}$, Jo Van Dorpe ${ }^{5}$, David Creytens $^{5}$ and Jean De Schepper ${ }^{1,6}$
}

\begin{abstract}
Case presentation: We report for the first time a synchronous papillary and follicular thyroid carcinoma in a 12year-old girl presenting with a large $(5 \mathrm{~cm}$ diameter) left thyroid nodule, an increased left and right upper pole technetium tracer uptake at scintigraphy and hyperthyroidism. The uptake at the right lobe was explained by the crossing of the left nodule to the right site of the neck at Computed Tomography (CT) scanning.

Background: Although thyroid nodules are less common in children than in adults, there is more vigilance required in children because of the higher risk of malignancy. According to literature, about $5 \%$ of the thyroid nodules in adults are malignant versus $20-26 \%$ in children. The characteristics of 9 other pediatric cases with a differentiated thyroid carcinoma presenting with a toxic nodule, which have been reported during the last 20 years, are summarized. A nodular size of more than $3.5 \mathrm{~cm}$ and female predominance was a common finding.

Conclusions: The presence of hyperthyroidism in association with a hyperfunctioning thyroid nodule does not rule out thyroid cancer and warrants careful evaluation, even in the absence of cervical lymph node invasion.
\end{abstract}

Keywords: Hyperthyroidism, Toxic nodule, Follicular thyroid carcinoma, Papillary thyroid carcinoma, Children, Pediatric

\section{Background}

Solitary thyroid nodules are rare in childhood in comparison with adulthood, but have a higher risk of malignancy [1]. Up to $26 \%$ of the thyroid nodules in children were found to be malignant [1-3]. Most differentiated thyroid cancers (DTC) in children are papillary carcinoma, which only very rarely (over) produce thyroid hormones, causing subclinical or overt hyperthyroidism [4]. In a recent pediatric cohort study of thyroid nodules, hyperthyroidism was found in only 5\% [5]. The finding of a hyperfunctioning or hot nodule on scintigraphy in

\footnotetext{
* Correspondence: Joke.VanVlaenderen@uzgent.be

'Department of Pediatric Endocrinology, University Hospital Ghent, Corneel Heymanslaan 10, 9000 Ghent, Belgium

Full list of author information is available at the end of the article
}

the context of hyperthyroidism, especially in the absence of enlarged cervical lymph nodes, is usually reassuring, as in most cases a benign follicular adenoma is diagnosed [5-7].

We additionally reviewed the previously reported DTC in children and adolescents with a hyperfunctioning thyroid nodule associated with hyperthyroidism, also called toxic nodule, to look for common characteristics and potential risk factors for malignancy. A literature search on Pubmed and Web of Science was performed in the English literature in a period from January 2009, when most laboratories were using a third generation TSH assay, until December 2019. The search terms used were "hyperthyroidism and thyroid carcinoma", "toxic nodule", "hyperfunctioning nodule and thyroid carcinoma" 
in conjunction with either "children" or "pediatric". Inclusion criteria were detailed case descriptions of children aged below 16 years old with a clinical and biological hyperthyroidism and an underlying differentiated thyroid carcinoma documented at histological examination. Finally, 8 articles describing 9 cases were selected. Clinical characteristics, biological, imaging and histological results and initial treatment of these 9 pediatric patients are summarized in Table 1.

\section{Overview of previously reported case reports of DTC in children with a toxic nodule [8-15]}

All described patients were female except one. Age at presentation varied between a minimum age of 2 months and a maximum age of 16 years (median 11 years). The severity of the hyperthyroidism is not always mentioned, but FT4 concentrations up to 3 times the upper limit with less elevated FT3 concentrations were reported. Right as well as left sided localisation of the nodule was seen, while the longest diameter of the nodule ranged from 35 to $50 \mathrm{~mm}$. Suppressed activity in surrounding tissue at scintigraphic evaluation was noted in 8 cases. In four cases a follicular variant of a papillary thyroid carcinoma was found, while in the others either a papillary thyroid carcinoma or a follicular carcinoma was present (Table 1).

\section{Case presentation}

In a 12-year-old female, presenting with a painless neck swelling since one month, a $5 \mathrm{~cm}$ long, non-tender but firm nodule in the left thyroid lobe was detected at physical examination. There was no palpable cervical lymphadenopathy. Slight tachycardia (pulse rate $95 / \mathrm{min}$ ), but no exophthalmos was present. The patient reported intermittent sore throat, increasing nervousness and a 2 $\mathrm{kg}$ weight loss in the last several months. There was no history of radiation exposure. Her mother had recently undergone surgery for a multi-nodular goitre. There was no family history of cancer or intestinal polyps.

Thyroid function tests showed an elevated FT4 (51 $\mathrm{pmol} / \mathrm{L}$ or $39.6 \mathrm{pg} / \mathrm{mL}$ ) and thyroglobulin concentration $(435 \mu \mathrm{g} / \mathrm{L})$ and a decreased TSH $(<0.005 \mathrm{mU} / \mathrm{L})$ concentration. ${ }^{1}$ Serum anti-thyroglobulin, anti-thyroidperoxidase and anti-TSH receptor antibodies were undetectable. Doppler ultrasound showed a normal right lobe as well as a sharp defined hypervascular solid multilobular mass (longest diameter $53 \mathrm{~mm}$ ) with cystic components with microcalcifications in the left lobe. A ${ }^{99 \mathrm{~m}}$ Technetium scintigraphy (Fig. 1) showed a global, but heterogeneous hyperfunctioning thyroid gland with excessive uptake at the upper left lobe and upper right lobe. A right tracheal

${ }^{1}$ Reference rate: FT4 $12.0-22.0 \mathrm{pmol} / \mathrm{L}$, thyroglobulin concentration $0.2-70.0 \mu \mathrm{g} / \mathrm{L}$, TSH $0.70-6.4 \mathrm{mU} / \mathrm{L}$ deviation by the left thyroid mass, but no cervical or mediastinal lymphnodes or lung masses, was seen on the CT scan of neck and thorax (Fig. 2). Subsequently, a large toxic adenoma crossing the midline was diagnosed, explaining the tracer uptake in the right upper pole region. Fine needle biopsy was refused by the patient and a left thyroidectomy was proposed. The patient underwent a left hemithyroidectomy after 2 months of methimazole therapy, which resulted in normalized thyroid function in one month's time. Histological examination of the left lobe showed two separated morphologically distinct neoplastic lesions arising in what appeared to be a multinodular goitre. The largest lesion (measuring $2.9 \mathrm{~cm}$ ) showed a follicular growth pattern with cytonuclear atypia, multiple foci of intracapsular vascular invasion and some foci of capsular invasion. Separated from this lesion by a couple of millimetres, a second neoplastic lesion was seen. This was non-encapsulated and infiltrative and had a papillary and follicular growth pattern with cytonuclear features of a papillary thyroid carcinoma (nuclear clearing, nuclear inclusions and grooves). The non-encapsulated, infiltrative neoplastic lesion with papillary architecture and cytomorphology showed aberrant apical and strong HBME immunohistochemical staining, whereas the adjacent lesion with follicular growth pattern was completely negative for HBME. Therefore, based on the morphology and immunohistochemistry, a diagnosis of a synchronous minimally invasive follicular thyroid carcinoma (with multifocal capsular invasion and angioinvasion) and a non-encapsulated, infiltrative (classical type) papillary thyroid carcinoma of the left lobe was made (Fig. 3). Consequently, total thyroidectomy was performed.

Genetic testing on the surgical specimen was negative for BRAF and NRAS mutations and RET/PTC rearrangements. Results of TSHR gene, GNAS gene and PTEN gene analysis were normal.

\section{Discussion}

Hyperfunctioning nodules at thyroid scintigraphy, also called hot nodules, can present with or without hyperthyroidism. In the latter case, these nodules are also described as toxic nodules in literature. In the previously reported nine pediatric cases of DTC with associated hyperthyroidism and a hot nodule at scintigraphy, follicular carcinomas, papillary carcinomas as well as follicular variants of papillary thyroid carcinoma were diagnosed. We report for the first time a synchronous papillary-follicular thyroid carcinoma in female adolescent presenting with a toxic nodule. In our case as well as in the previously reported pediatric cases, the nodules were found to be greater than $3 \mathrm{~cm}$ in diameter, suggesting that clinical hyperthyroidism does not appear until the nodule is at least $3 \mathrm{~cm}$ in diameter. When comparing with non-hyperfunctioning nodules, thyroid nodules in hyperthyroid adolescents were 


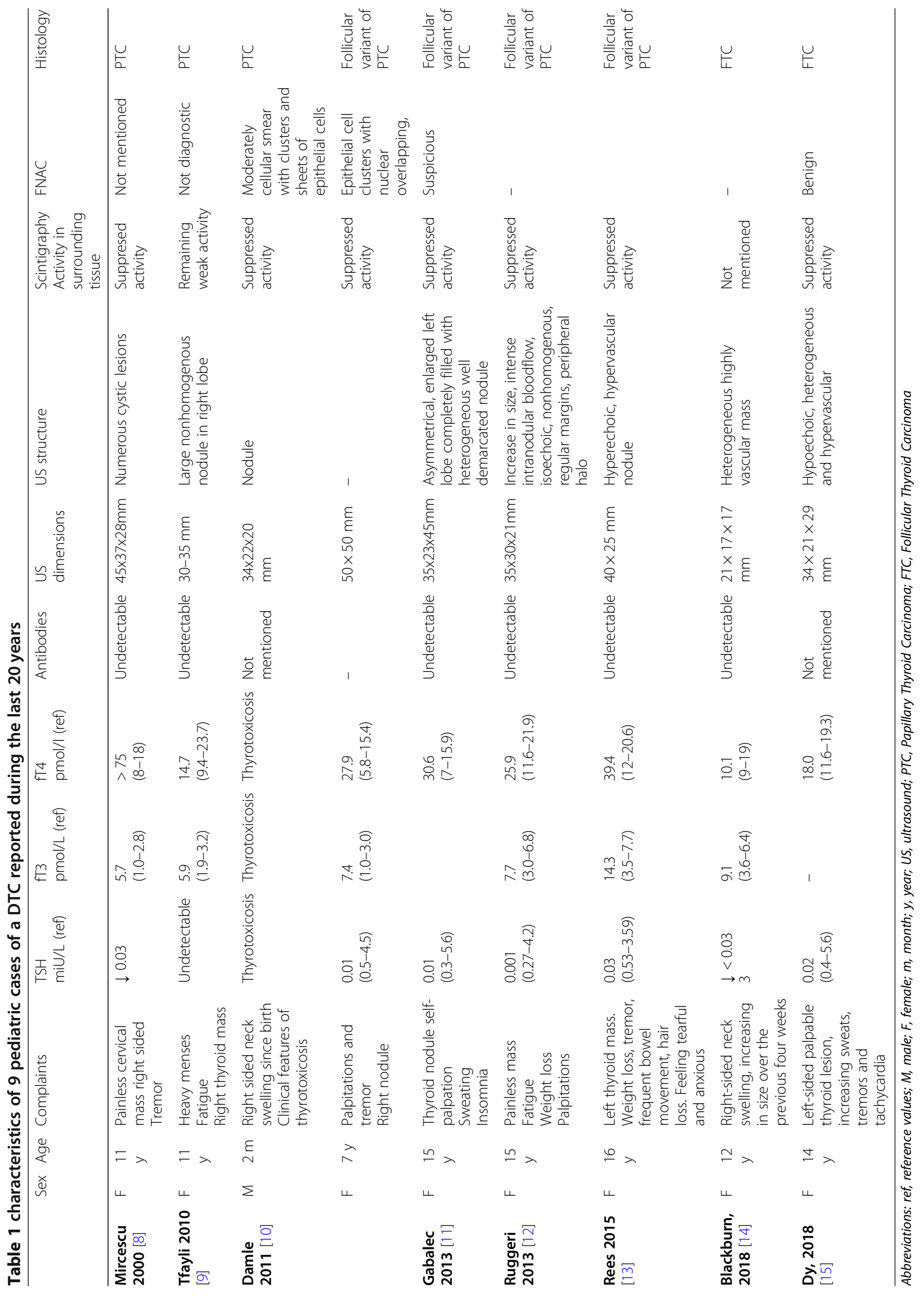




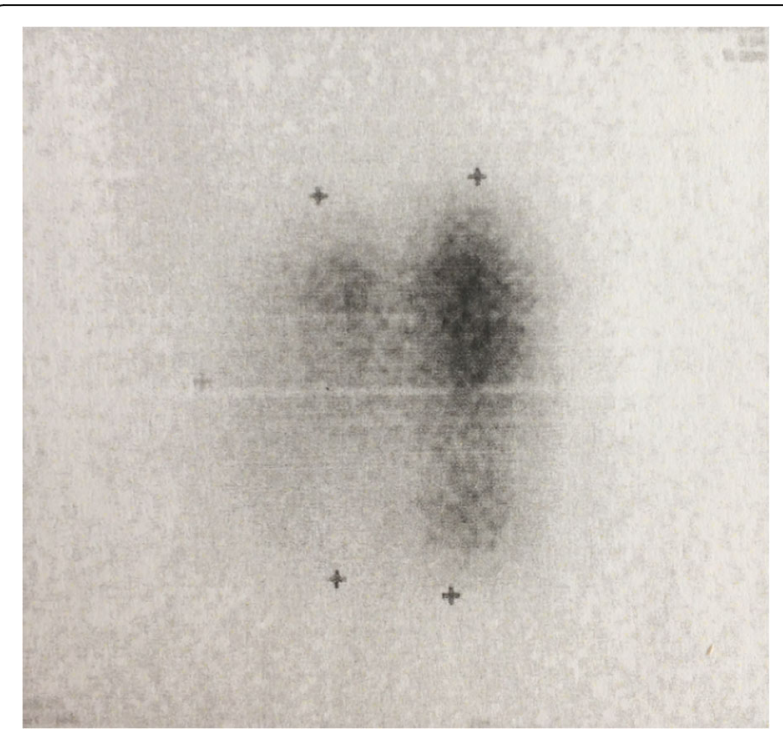

Fig. 1 Scintigraphic image showing a global but heterogeneous hyperfunctioning thyroid gland with excessive uptake at upper left lobe and upper right lobe

found to have more compressive signs and a greater nodule size, and are mostly diagnosed as follicular adenomas (toxic adenoma) $[5,16]$.

The major goal of the diagnostic evaluation of thyroid nodules is to differentiate thyroid cancers, especially aggressive lesions, from benign adenomas. In the initial work-up of a thyroid nodular lesion, thyroid function tests are usually performed. The American Thyroid Association (ATA) Taskforce recommends that patients who have a thyroid nodule larger than 1 to $1.5 \mathrm{~cm}$ in any dimension, should have a serum thyrotropin (TSH) measurement [17]. If hyperthyroidism is associated with a nodule on ultrasound, a scintiscan is the next logical

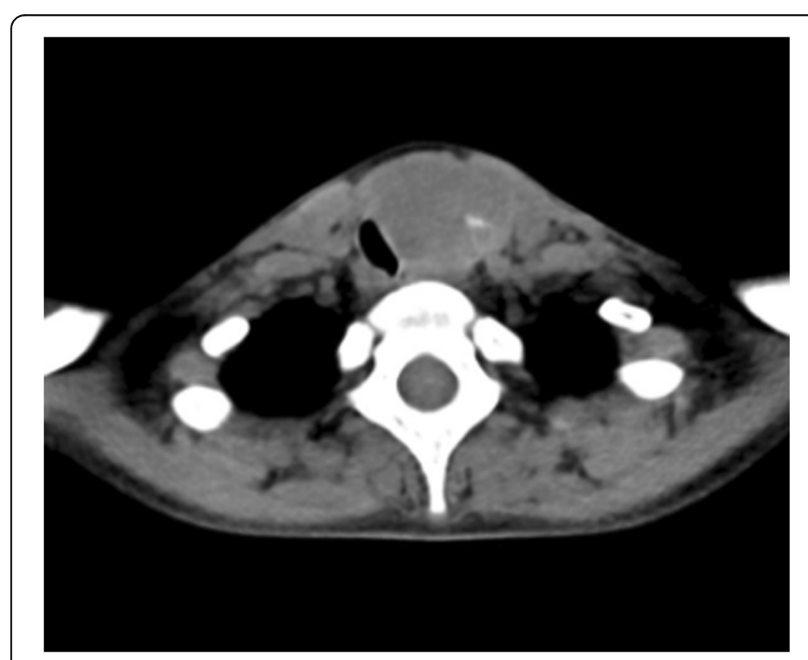

Fig. 2 CT image showing a right tracheal deviation by a left thyroid mass step to document the hyperfunctioning of the nodule, especially when thyroid stimulating immunoglobulines are absent. In toxic adenoma, the typical scintigraphic finding is a hot pattern in the nodule with the remnant thyroid tissue showing a severely decreased or absent uptake [18]. In our case no complete suppression was found, while in the other pediatric cases both complete and incomplete scintigraphic suppression patterns were reported. An incomplete suppression pattern was seen as a risk factor for DTC by Niedziela et al. [19] in his series of 31 children with a hyperfunctioning nodule.

The prevalence of malignancy in a hot nodule in adults has been estimated at 3.1\% [20]. Histological outcome studies in children with a toxic nodule are very limited. No malignancy was detected in 6 Italian hyperthyroid pediatric patients with a solitary toxic nodule at surgery [5]. In an American study of 4 children with a hot or warm nodule and persisting T3 hyperthyroidism, no malignancy was found after partial thyroidectomy [18], while in another study of 2 hyperthyroid adolescents a follicular carcinoma was found in one female [21]. However, in a more recent study of 15 Polish children with hyperthyroidism and a hyperfunctioning nodule at scintigraphy, a DCT was diagnosed in 2 children after surgery [22]. In none of the reported adult or pediatric cases a simultaneous papillary and follicular carcinoma in a hot nodule was described. The simultaneous occurrence of different types of thyroid cancer in a single patient is very rare. Although there are noticeable reports about synchronous papillary cancer, the reports of simultaneous papillary and follicular cancer are actually rare [23]. This simultaneous thyroid tumor presentation has been described as coincidental in the literature as no common gene mutation for the pathogenesis of the different tumor types of the thyroid has been found. Mixed tumors however can occur as part of familial cancer syndromes. Cowden's syndrome was excluded in our patient by genetic analysis, while no signs of Carney's complex were present. Fine needle aspiration (FNA) biopsy is considered to be the most accurate procedure to identify malignant nodules, but is generally not advised in hot nodules. First, their larger size might easily result in false negative results. Second, hot nodules have lower likelihood of finding malignancy compared to cold nodules and third, there are histological difficulties in differentiating follicular adenoma from follicular thyroid carcinoma.

Contrary to guidelines for adults, the current recommendation for treatment of hyperfunctioning nodules in children is surgical resection rather than radio iodine treatment $[11,24]$. Surgery is even more preferred when there are signs of compression, which is a common finding in hyperfunctioning nodules since they are larger in most cases than non-functional nodules [25]. Because of the absence of clinical (male gender, age $<10$ years, family history of thyroid cancer) and ultrasound (irregular margins) risk factors, as 


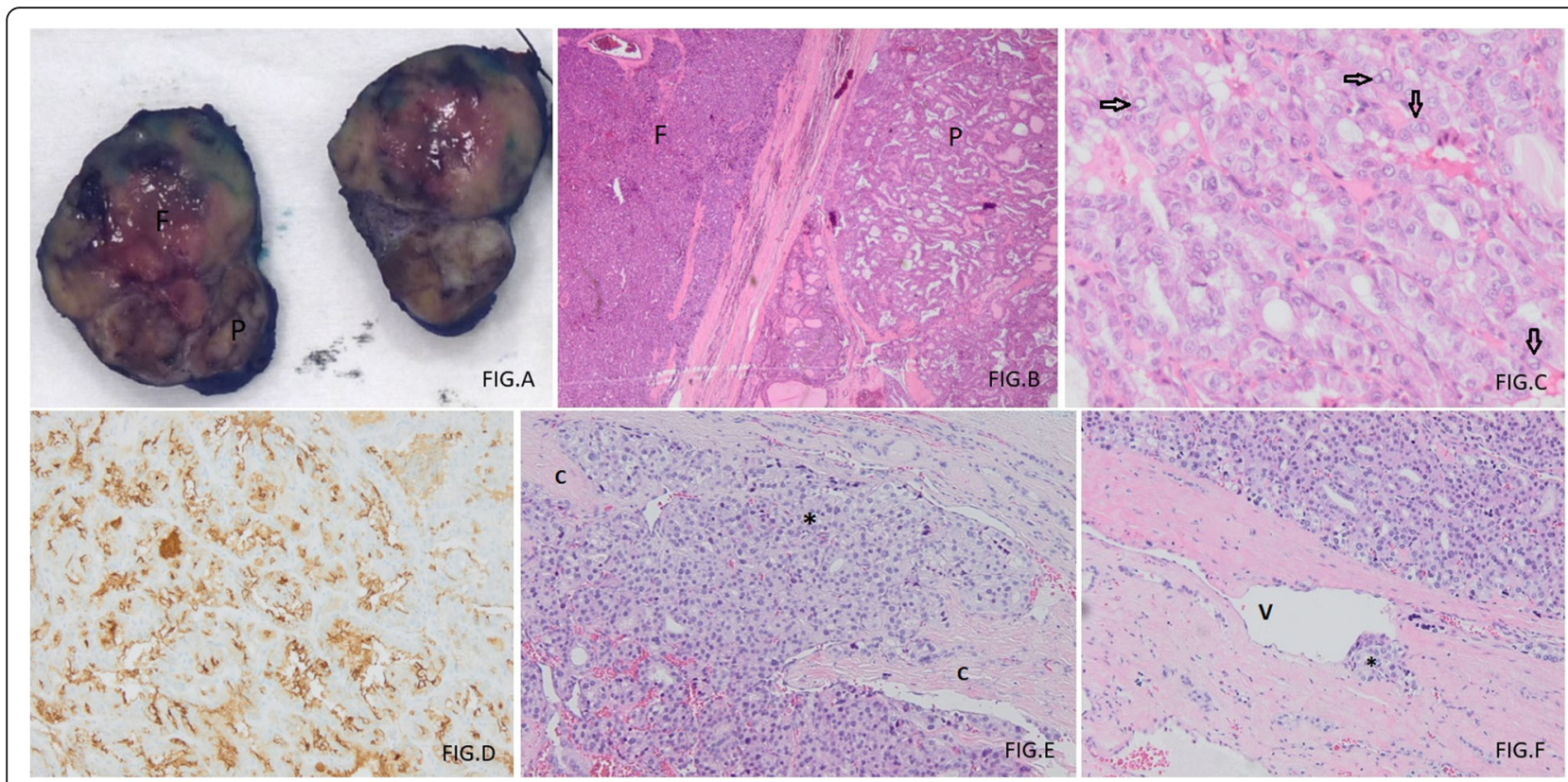

Fig. 3 a Gross pathology (sections). Two separated neoplastic tumors in the left thyroid lobe: follicular thyroid carcinoma (F) (at the top) and papillary thyroid carcinoma (P) (at the bottom). b Two separated morphologically distinct neoplastic tumors in the left thyroid lobe: minimally invasive follicular thyroid carcinoma (F) (to the left) and papillary thyroid carcinoma (P) (to the right) (Hematoxylin and Eosin, original magnification 40x). c Cytonuclear features of papillary thyroid carcinoma, including nuclear overlapping, nuclear grooves ( $\downarrow$ ) and nuclear clearing $(\rightarrow)$ (Hematoxylin and Eosin, original magnification 200x). d Aberrant strong apical membranous (brown coloured) HBME expression in the papillary thyroid carcinoma (original magnification 200x). e Capsular invasion (C) in the follicular thyroid carcinoma $\left(^{*}\right.$ ) (Hematoxylin and Eosin, original magnification 100x). f Angioinvasive growth $(V)$ in the follicular thyroid carcinoma ${ }^{*}$ ) (Hematoxylin and Eosin, original magnification 100x)

well as the absence of enlarged cervical lymph nodes, mediastinal and lung invasion at CT scanning, DCT was initially not suspected in this case and a hemithyroidectomy was performed with a the preoperative diagnosis of a large toxic adenoma. In the presence of a size of more than $4 \mathrm{~cm}$ and an intense internal vascularisation (and calcifications in the nodule), an initial total thyroidectomy could have been justified, as suggested by Deluca et al. [26]. On the other hand, a family history of multinodular goitre and the absence of cervical and lung invasion was assessed as a reassuring feature in our case, favouring a left hemithyroidectomy. We first treated our patient with methimazole, as patients with overt hyperthyroidism due to a hyperfunctioning nodule should be euthyroid prior to the surgical procedure [25]. In all reported cases, euthyroidism was observed relatively quickly after medical treatment, as observed in our case.

Somatic mutations of the TSHR and GNAS1 gene have been detected in adolescents presenting with autonomous functioning benign thyroid nodule as well as thyroid carcinoma with or without associated hyperthyroidism [8, 14, 22]. In one study, in 17 of 29 of benign hyperfunctioning nodules somatic TSHR mutations were found, while only one of the 4 studied DCT a mutation was found. Surgical specimen examination in our case was negative for BRAF and NRAS mutations and RET/ $P T C$ rearrangements. Increased malignancy rate of hyperfunctioning nodules was found not to be associated with BRAF, NRAS mutations and PAX8/PPARG and $R E T / P T C$ rearrangements [22].

\section{Conclusion}

In conclusion, our case illustrates the difficulty to accurately determine the risk of DTC in hyperthyroid adolescents presenting with a toxic nodule and provides histological evidence that a large thyroid carcinoma can be composed of both a follicular and papillary carcinoma.

\section{Abbreviations \\ ATA: American Thyroid Association; CT: Computed Tomography; DTC: Differentiated Thyroid Cancer; FT3: Free Triiodothyonine; FT4: Free Thyroxine; TSH: Thyroid Stimulating Hormone}

\section{Acknowledgements}

Not applicable.

\section{Authors' contributions}

$\mathrm{KL}$ was the general pediatrician, subsequently referral to KDW (pediatric endocrinology). After that, JDS followed the patient clinically. HV and WH did the surgical aspect. ES interpreted the radiologic imaging. HVB, JVD and DC performed the histological examination of the thyroid. HVB was responsible for the histological images. DC reviewed more extensive the histological part of the manuscript. JW and JDS were the major contributors in writing the manuscript. All authors read and approved the final manuscript.

\section{Funding}

No funding to report. 


\section{Availability of data and materials}

Data sharing is not applicable to this article as no datasets were generated or analysed during the current study.

\section{Ethics approval and consent to participate}

Case report, no need for ethics approval.

\section{Consent for publication}

Present.

\section{Competing interests}

The authors declare that they have no competing interests.

\section{Author details}

${ }^{1}$ Department of Pediatric Endocrinology, University Hospital Ghent, Corneel Heymanslaan 10, 9000 Ghent, Belgium. ${ }^{2}$ Department of Pediatrics, AZ Delta, Roeselare, Brugsesteenweg 90, 8800 Roeselare, Belgium. ${ }^{3}$ Department of Radiology, University Hospital Ghent, Corneel Heymanslaan 10, 9000 Ghent, Belgium. ${ }^{4}$ Department of Head and Neck Surgery, University Hospital Ghent, Corneel Heymanslaan 10, 9000 Ghent, Belgium. ${ }^{5}$ Department of Pathology, University Hospital Ghent, Corneel Heymanslaan 10, 9000 Ghent, Belgium. ${ }^{6}$ Department of Pediatric Endocrinology, University Hospital Brussels, Laarbeeklaan 101, 1090 Brussels, Belgium.

Received: 25 March 2020 Accepted: 18 June 2020

Published online: 21 July 2020

\section{References}

1. Niedziela M. Pathogenesis, diagnosis and management of thyroid nodules in children. Endocr Relat Cancer. 2006;13(2):427-53.

2. Divarci E, Celtik U, Dokumcu Z, Ergun O, Ozok G, Ozen S, et al. Management of Childhood Thyroid Nodules: surgical and Endocrinological findings in a large Group of Cases. J Clin Res Pediatric Endocrinol. 2017;9(3):222-8.

3. Hung W. Solitary thyroid nodules in 93 children and adolescents. A 35-years experience. Horm Res. 1999;52(1):15-8.

4. Hesselink MSK, Nies M, Bocca G, Brouwers AH, Burgerhof JGM, van Dam EWCM, et al. Pediatric differentiated thyroid carcinoma in the Netherlands: a Nationwide follow-up study. J Clin Endocr Metab. 2016;101(5):2031-9.

5. Corrias A, Mussa A, Baronio F, Arrigo T, Salerno M, Segni M, et al. Diagnostic features of thyroid nodules in pediatrics. Arch Pediat Adol Med. 2010;164(8): 714-9.

6. Hung W, August GP, Randolph JG, Schisgall RM, Chandra R. Solitary thyroid nodules in children and adolescents. J Pediatr Surg. 1982:17(3):225-9.

7. Boelaert K, Horacek J, Holder RL, Watkinson JC, Sheppard MC, Franklyn JA. Serum thyrotropin concentration as a novel predictor of malignancy in thyroid nodules investigated by fine-needle aspiration. J Clin Endocrinol Metab. 2006:91(11):4295-301.

8. Mircescu H, Parma J, Huot C, Deal C, Oligny LL, Vassart G, et al. Hyperfunctioning malignant thyroid nodule in an 11-year-old girl: pathologic and molecular studies. J Pediatr. 2000;137(4):585-7.

9. Tfayli HM, Teot LA, Indyk JA, Witchel SF. Papillary thyroid carcinoma in an autonomous hyperfunctioning thyroid nodule: case report and review of the literature. Thyroid. 2010;20(9):1029-32.

10. Damle N, Gupta S, Kumar P, Mathur S, Bal C. Papillary carcinoma masquerading as clinically toxic adenoma in very young children. J Pediatric Endocrinol Metabolism. 2011;24(11-12):1051-4.

11. Gabalec F, Svilias I, Plasilova I, Hovorkova E, Ryska A, Horacek J. Follicular variant of papillary carcinoma presenting as a hyperfunctioning thyroid nodule. J Pediatr Hematol Oncol. 2014;36(2):e94-6.

12. Ruggeri RM, Campenni A, Giovinazzo S, Saraceno G, Vicchio TM, Carlotta D, et al. Follicular variant of papillary thyroid carcinoma presenting as toxic nodule in an adolescent: coexistent polymorphism of the TSHR and Gsalpha genes. Thyroid. 2013;23(2):239-42.

13. Rees DO, Anthony VA, Jones K, Stephens JW. Follicular variant of papillary thyroid carcinoma: an unusual cause of thyrotoxicosis. BMJ Case Rep. 2015; 2015.

14. Blackburn J, Giri D, Ciolka B, Gossan N, Didi M, Kokai G, et al. A rare case of heterozygous gain of function Thyrotropin receptor mutation associated with development of thyroid follicular carcinoma. Case Reports in Genet. 2018;2018:1381730.
15. Dy BM, Katabi N, Boucai L, Shaha A. Follicular carcinoma masquerading as a hot nodule in a pediatric patient. Am Surgeon. 2018;84(6):1117-9.

16. Molnar GD, Wilber RD, Lee RE, Woolner LB, Keating FR Jr. On the Hyperfunctioning solitary thyroid nodule. Mayo Clin Proc. 1965:40:665-84.

17. Cooper DS, Doherty GM, Haugen BR, Kloos RT, Lee SL, Mandel SJ, et al. Management guidelines for patients with thyroid nodules and differentiated thyroid cancer. Thyroid. 2006;16(2):109-42.

18. Osburne RC, Goren EN, Bybee DE, Johnsonbaugh RE. Autonomous thyroidnodules in adolescents - clinical characteristics and results of Trh testing. J Pediatr-Us. 1982;100(3):383-6.

19. Niedziela M, Breborowicz D, Trejster E, Korman E. Hot nodules in children and adolescents in western Poland from 1996 to 2000: clinical analysis of 31 patients. J Pediatric Endocrinol Metabolism. 2002;15(6):823-30.

20. Mirfakhraee S, Mathews D, Peng L, Woodruff S, Zigman JM. A solitary hyperfunctioning thyroid nodule harboring thyroid carcinoma: review of the literature. Thyroid Res. 2013;6(1):7.

21. Croom RD 3rd, Thomas CG Jr, Reddick RL, Tawil MT. Autonomously functioning thyroid nodules in childhood and adolescence. Surgery. 1987; 102(6):1101-8.

22. Eszlinger M, Niedziela M, Typlt E, Jaeschke H, Huth S, Schaarschmidt J, et al. Somatic mutations in 33 benign and malignant hot thyroid nodules in children and adolescents. Mol Cell Endocrinol. 2014:393(1-2):39-45.

23. Cracolici V, Mujacic I, Kadri S, Alikhan M, Niu N, Segal JP, et al. Synchronous and metastatic papillary and follicular thyroid carcinomas with unique molecular signatures. Endocr Pathol. 2018;29(1):9-14.

24. Francis GL, Waquespack SG, Bauer AJ, Angelos P, Benvenga S, Cerutti JM, et al. Management guidelines for children with thyroid nodules and differentiated thyroid Cancer. Thyroid. 2015;25(7):716-59.

25. Ross DS, Burch HB, Cooper DS, Greenlee MC, Laurberg P, Maia AL, et al. 2016 American Thyroid Association guidelines for diagnosis and Management of Hyperthyroidism and Other Causes of thyrotoxicosis. Thyroid. 2016;26(10):1343-421.

26. Deluca F, Chaussain JL, Job JC. Hyperfunctioning thyroid-nodules in children and adolescents. Acta Paediatr Scand. 1986;75(1):118-23.

\section{Publisher's Note}

Springer Nature remains neutral with regard to jurisdictional claims in published maps and institutional affiliations.

Ready to submit your research? Choose BMC and benefit from:

- fast, convenient online submission

- thorough peer review by experienced researchers in your field

- rapid publication on acceptance

- support for research data, including large and complex data types

- gold Open Access which fosters wider collaboration and increased citations

- maximum visibility for your research: over $100 \mathrm{M}$ website views per year

At $\mathrm{BMC}$, research is always in progress.

Learn more biomedcentral.com/submissions 